\title{
Early prolonged ambulatory cardiac monitoring in stroke (EPACS): an open-label randomised controlled trial
}

\author{
Amit Kaura ${ }^{1,2^{*}} \mathbb{0}$, Laszlo Sztriha ${ }^{1}$, Fong Kum Chan ${ }^{1}$, John Aeron-Thomas ${ }^{1}$, Nicholas Gall' \\ Bartlomiej Piechowski-Jozwiak and James T. Teo ${ }^{1}$
}

\begin{abstract}
Background: Cardioembolism in paroxysmal atrial fibrillation (PAF) is a preventable cause of transient ischaemic attack (TIA) or ischaemic stroke; however, due to its transient nature, a short-duration Holter monitor may miss a significant proportion of events.

Methods: We conducted an open-label randomised controlled trial of cardiac monitoring after a TIA or ischaemic stroke comparing a 14-day ECG monitoring patch (Zio ${ }^{\circledR}$ Patch, iRhythm Technologies) with short-duration Holter monitoring for the detection of PAF. The primary outcome was the detection of one or more episodes of ECG-documented PAF lasting at least $30 \mathrm{~s}$ within 90 days in each of the study arms. A budget impact analysis from the healthcare perspective was performed.

Results: From February 2016 through February 2017, 43 (76.8\%) of the 56 patients assigned to the patch-based monitoring group and 47 (78.3\%) of the 60 patients assigned to short-duration Holter monitoring group had successful monitor placement with 90 days of follow-up. Of the 26 protocol failures between the two groups, 23 (88.5\%) were due to patient refusal for outpatient short-duration ECG monitor placement, whilst only 1 (3.8\%) was due unsuccessful ZioPatch placement. The rate of detection of PAF at 90 days was $16.3 \%$ in the patch-based monitoring group (seven patients) compared to $2.1 \%$ in the short-duration Holter monitoring group (1 patient), with an odds ratio of 8.9 (95\% Cl 1.1-76.0; $P=0.026$ ). An economic model demonstrated that implementation of the Zio Patch service would result in 10.8 more strokes avoided per year compared to current practice with Holter monitoring with an associated yearly saving in direct medical costs of $£ 113,630$, increasing to $£ 162,491$ over 5 years.
\end{abstract}

Conclusions: Early, prolonged, patch-based monitoring after an index stroke or TIA is superior to short-duration Holter monitoring in the detection of PAF and likely cost-effective for preventing recurrent strokes.

Trial registration http://www.isrctn.com. Unique identifier: ISRCTN 50253271. Registered 21 January 2016

Keywords: Atrial fibrillation, Cardiac monitoring, Electrocardiography, Ischaemic stroke, Medical devices

\footnotetext{
*Correspondence: amit.kaura@nhs.net

${ }^{1}$ King's College London NHS Foundation Trust, King's College Hospital,

Denmark Hill, London SE5 9RS, UK

Full list of author information is available at the end of the article
} 


\section{Background}

A frequent cause of ischaemic stroke or transient ischaemic attack (TIA) is due to cardioembolism in atrial fibrillation (AF), but up to $40 \%$ of ischaemic strokes are classified as cryptogenic, with no cause identified after routine evaluation $[1,2]$. This is because AF is often paroxysmal (paroxysmal atrial fibrillation, PAF) and a single 12-lead ECG or even 24-h Holter ECG monitoring may miss a significant proportion of patients with PAF [3]. While stroke guidelines from both North America and the UK suggest that more prolonged cardiac rhythm monitoring may be indicated, there is currently no consensus on the recommended duration of ECG monitoring $[4,5]$.

Available approaches for ECG monitoring beyond $24 \mathrm{~h}$ have their limitations. While continuous bedside ECG monitoring during an approximately 3-day inpatient stay on the stroke unit has a significantly higher sensitivity in detecting PAF than 24-h Holter monitoring (7.7\% vs $2.8 \%$ ) [6], this approach is not suitable for outpatients who are ambulant immediately following their stroke. While systems for recording beyond 3-7 days have demonstrated even higher detection rates of PAF $[7,8]$, they have significant limitations: event-triggered loop recorders are cumbersome while implanted loop recorders require a minor surgical procedure. There is therefore a need for a patient-friendly, long-duration, ECG monitoring system for patients with cryptogenic stroke or TIA. The ZioPatch ${ }^{\circledR}$ (iRhthym Technologies, USA) is a novel, adhesive cardiac monitoring patch which provides an alternative method for prolonged ECG monitoring for the detection of PAF [9-11]. This waterproof patch is applied non-invasively to the anterior chest wall for continuous monitoring for up to 14 days without requiring any complex setup. The ECG trace uses the Zio XT algorithmic support to highlight areas for human interpretation.

We conducted a pragmatic randomised controlled trial of cardiac monitoring for the detection of PAF, in patients with cryptogenic stroke or TIA, using the patchbased monitor or a standard short-duration Holter monitor. We hypothesised that early prolonged ambulatory cardiac monitoring would enhance the detection of PAF in patients who would be candidates for anticoagulation therapy for secondary stroke prevention.

\section{Methods}

\section{Study design}

The early prolonged ambulatory cardiac monitoring in stroke (EPACS) study was a prospective randomised open-blinded endpoint trial comparing the efficiency of PAF detection with the 14-day patch-based monitor versus conventional medical therapy of short-duration
Holter ECG in patients with cryptogenic ischaemic stroke or TIA early after the index event. With a sample size of 120 , the study was powered at $90 \%$ (beta set at 0.10 ), based on a conservative effect size from previous studies (EMBRACE [8] and CRYSTAL-AF [7]). A single interim analysis at a sample size of 40 participants was performed using O'Brien-Fleming stopping boundaries to maintain an overall alpha level of 0.05 .

The study protocol is summarised in Fig. 1. Patients were randomly assigned in a 1:1 ratio to one of the two ECG monitoring strategies using a computerised block randomisation generator provided externally by the King's Clinical Trials Unit, stratified for age, gender and history of hypertension. Patients were enrolled from King's College Hospital NHS Foundation Trust, UK, across two sites, King's College Hospital, an urban teaching hospital, and Princess Royal University Hospital, a suburban district general hospital.

All patients were provided with verbal and written information about the trial, and informed written consent was obtained before enrolment. The trial was registered with The National Research Ethics Service and the local Research and Development Committee approved the study protocol. The Medicines and Healthcare products Regulatory Agency verified EPACS as a post-marketing study which did not require specific authorisation. The trial was registered on the International Standard Randomised Controlled Trial Number Register (ISRCTN 50253271).

The trial was conducted in accordance with the principles of the Declaration of Helsinki and the guidelines for Good Clinical Practice (EMA/CPMP/CIH/135/1995).

The funder (Bristol-Meyers-Squibb) provided financial support for this project and had no influence in the conduct of the study or the drafting of the analysis. The manufacturer of the ZioPatch cardiac monitor (iRhythm Technologies, USA) was not involved in the study design, conduct of trial or in the drafting of this paper; iRhythm provided support for inventory management and remote data management of devices.

\section{Patient eligibility}

Eligible patients were 18 years of age or older and were diagnosed with having had an ischaemic non-lacunar stroke or TIA within the past $72 \mathrm{~h}$ by a stroke physician or neurologist. Patients with a TIA were enrolled only if there were cortical symptoms of hemianopia or dysphasia at presentation or if their diffusion-weighted cerebral MRI scan was positive in a non-lacunar distribution. The main exclusion criteria were a history of AF or atrial flutter, carotid stenosis $>50 \%$, a pre-existing indication or contraindication for permanent anticoagulation therapy or those unable to provide informed consent. 


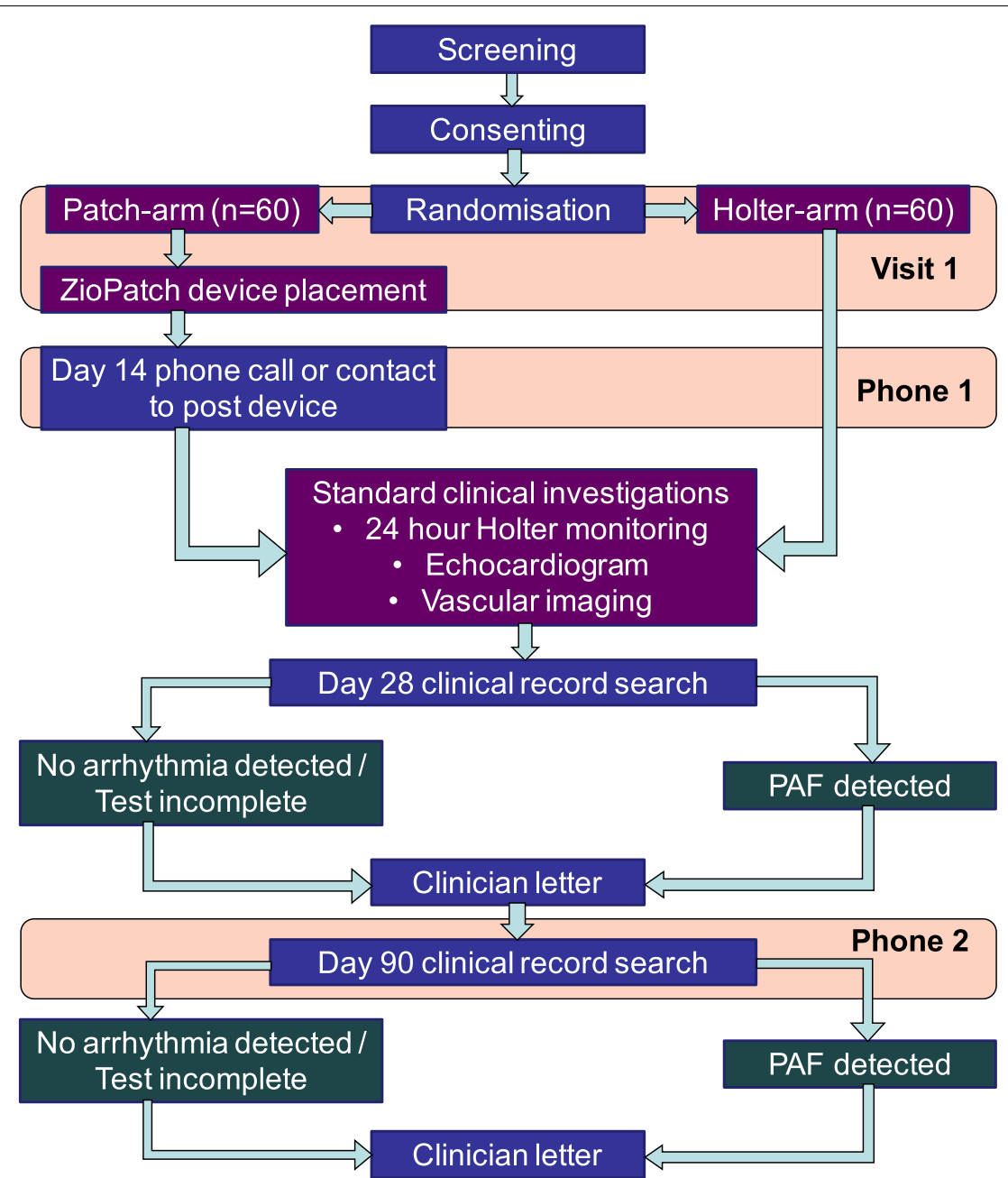

Fig. 1 Flowchart of trial protocol. PAF paroxysmal atrial fibrillation

\section{ECG monitoring strategies}

Patients assigned to the conventional medical therapy arm received current medical therapy of ambulatory Holter monitoring only (duration determined by treating physician, which was usually $24 \mathrm{~h}$ ), either arranged as an inpatient or outpatient depending on the anticipated duration of inpatient stay as per hospital protocol. Patients assigned to the patch-based monitoring arm had the patch applied to the anterior chest wall with the device kept in-situ for 14-days continuously, removed only for short intervals, such as during MRI imaging. Patients discharged from hospital prior to day 14 were discharged with the patch in-situ. On day 14, patients received a phone call reminder to return the device in a postage-paid envelope (Fig. 1). The patch was directly received by the manufacturer and the data retrieved and processed by the company's Zio ECG Utilisation Service. The summary report was subsequently accessed via a secure website and the reports reviewed by a cardiologist for report verification following review of the rhythm strips provided. Patients randomised to the patch-based monitoring arm also had standard practice of short-duration Holter monitoring. The Lifecard CF Holter Recorder (Spacelabs Healthcare, USA) was used for the Holter ECG. All ECG traces supplied from the short-duration Holter monitor and patch-based monitor were assessed by an experienced cardiologist; the patch-based monitor used an automated algorithmic support to highlight areas for diagnostic interpretation. Patients in both study arms were followed up for 90 days, without direct contact by the research team.

\section{Follow-up and clinical outcomes}

At day 28 and at 90, we conducted an electronic hospital medical records data search and phone call to the patient's general practitioner in the community to collect endpoint data (Fig. 1). The primary outcome was the detection of one or more episodes of ECG-documented 
PAF lasting at least $30 \mathrm{~s}$ within 90 days in each of the study arms (inter-subject comparison). This included PAF documented on the ECG monitoring strategies or detected incidentally during usual clinical practice, such as during echocardiography.

Secondary outcomes included PAF lasting at least $30 \mathrm{~s}$ within 28 days in each of the study arms and PAF lasting at least $30 \mathrm{~s}$ detected on the patch-based monitoring or short-duration Holter monitor within 90 days in patients who underwent both ECG monitoring strategies (intra-subject comparison). Other secondary outcomes included anticoagulation therapy use at day 90 , the proportion of patients with ischaemic stroke or TIA at day 90, mortality at day 90 and time-to-reporting of ECG monitoring. Decisions on patient management (including anticoagulation) were made by the patient's responsible physician and not the research team.

\section{Economic evaluation}

Formal cost-effectiveness assessment of new diagnostic devices is desirable. However, the current study yields insufficient information on clinical outcomes to support a cost-utility model, given the limited 90-day follow-up period. This process will need to be undertaken as more data become available in the future. As an indicative exercise, a budget impact analysis from the healthcare perspective was carried out by Imperial College Health Partners to assess the theoretical economic implications of the Zio Patch service versus Holter monitoring. Based on the AF detection rates found in this study, Hospital Episode Statistics data for the incidence of stroke and TIA (October 2016-September 2017) [12] and NHS reference costs [13], the cost-effectiveness of the ZioPatch service versus Holter monitoring at King's College Hospital NHS Foundation Trust was calculated. The Sentinel Stroke National Audit Programme estimate of $£ 13,452$ was used as the mean year 1 direct medical cost of a stroke [14]. All probability and cost assumptions made for the economic model can be reviewed in Additional file 1 .

\section{Statistical analyses}

Per-protocol analyses were performed for all comparisons of outcome as intention-to-treat was not balanced due to the high drop-out rate for Holter ECG's. Categorical variables were compared using the Fisher's Exact test. The $t$ test was used to compare continuous variables that were approximately normally distributed and the Wilcoxon Mann-Whitney Rank-Sum test was used for continuous variables that were not normally distributed. Statistical significance was set at $P<0.05$ (2-sided). SPSS (version 21.0, SPSS Inc., Chicago, USA) was used for the statistical analyses.

\section{Results}

\section{Study population}

From February 2016, through to February 2017, a total of 129 patients were recruited and 116 were randomly assigned to either the patch-based monitoring group (56 patients) or the short-duration Holter monitoring group (60 patients) (Fig. 2). The mean $( \pm S D)$ time between the index event and randomisation was $2.0 \pm 1.2$ days. Of the 56 patients assigned to the ZioPatch group, 43 (76.8\%) completed 90 days of follow-up with successful patch-based monitoring and short-duration Holter monitor recordings. Forty-seven (78.3\%) of the 60 patients assigned to short-duration Holter monitoring had successful monitor placement with 90 days of follow-up. Of the 26 protocol failures between the two groups, $23(88.5 \%)$ were due to patient refusal for outpatient short-duration ECG monitor placement, whilst only $1(3.8 \%)$ was due unsuccessful ZioPatch placement. The mean time from stroke or TIA to device placement was $2.1 \pm 1.2$ days and $38.9 \pm 33.6$ days with the ZioPatch and short-duration monitor, respectively. The mean wear time for the 14-day patch-based monitor and short-duration Holter monitor was $283.8 \pm 88.7 \mathrm{~h}$ and $25.0 \pm 25.0 \mathrm{~h}$, respectively. Patch-based monitoring was completed successfully in 54 out of 56 patients (96.4\%) with the majority completing the full 14-days of recording.

Baseline characteristics of the randomly assigned patients are shown in Table 1 . The mean age was $70.4 \pm 13.2$ years, $38.9 \%$ of patients were women, and $85.6 \%$ of index events were classified as a stroke. The mean $\mathrm{CHADS}_{2} \mathrm{VASc}_{2}$ score of all patients was $4.3 \pm 1.0$ points. None of the patients had previously received anticoagulation therapy or had a previous diagnosis of AF.

\section{Primary endpoint}

The patch-based monitoring strategy was superior to short-duration Holter monitoring for the detection of PAF lasting $30 \mathrm{~s}$ or longer-the rate of detection of PAF at 90 days was $16.3 \%$ among patients assigned to the ZioPatch patch-based monitoring group (seven patients), as compared to $2.1 \%$ among patients assigned to the shortduration Holter monitoring group (one patient), with an odds ratio of 8.9 (95\% confidence interval (CI) 1.1-76.0; $P=0.026$ ) (Table 2). One of the seven patients who had PAF in the patch-based monitoring group had PAF detected on the short-duration Holter monitor recording alone.

\section{Secondary endpoints}

The rate of detection of PAF at 28 days was $14.0 \%$ (6 patients) in the patch-based monitoring group, as compared to $2.1 \%$ (1 patient) in the short-duration Holter 


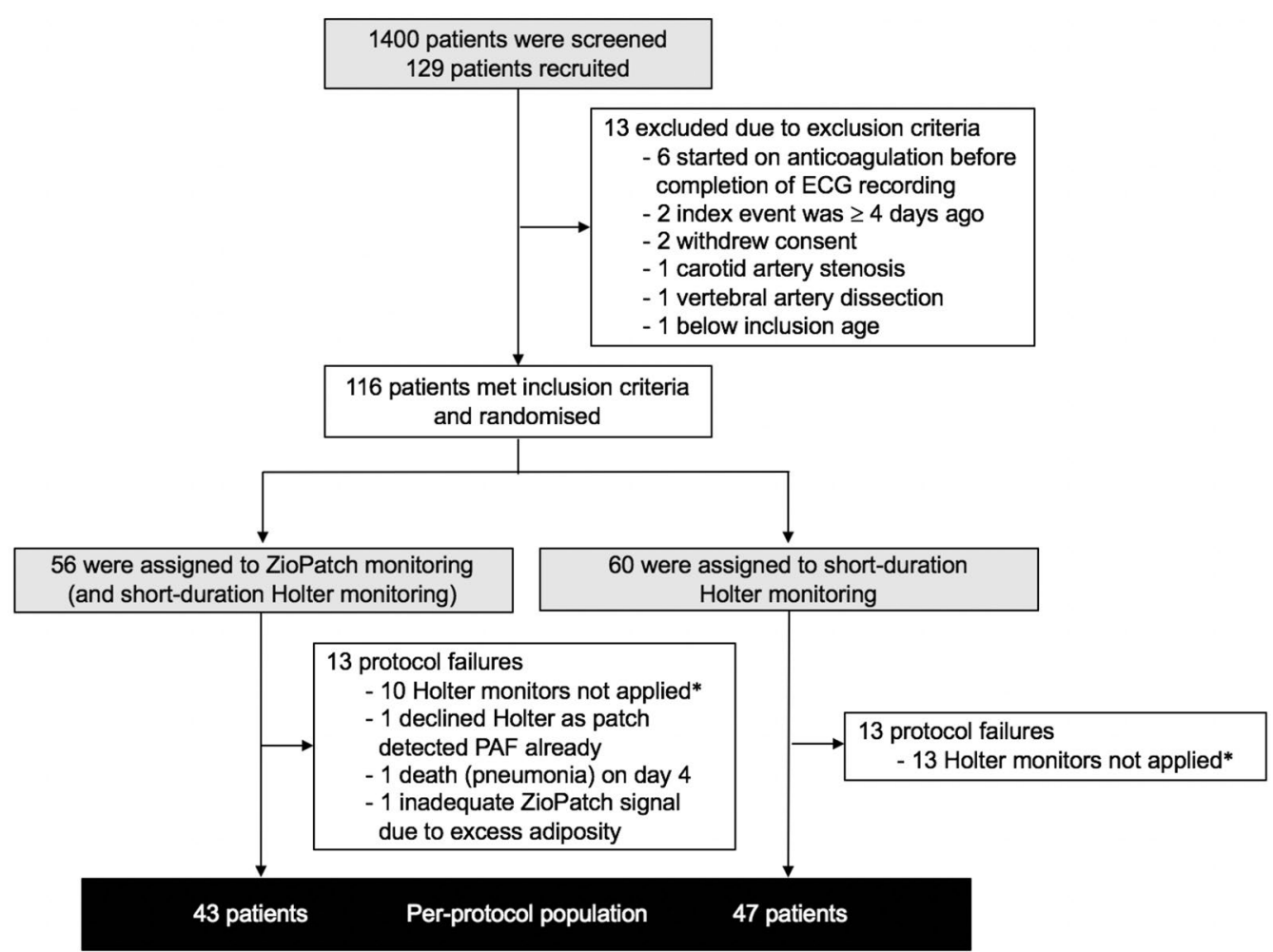

Fig. 2 Flowchart of enrolment, randomisation and follow-up of study participants. ECG electrocardiography, PAF paroxysmal atrial fibrillation. *Patient refusal for outpatient short-duration ECG monitor placement

monitoring group (odds ratio 7.5; 95\% CI 0.9-64.7; $P=0.05$ ) (Table 2). In the patch-based monitoring group, the detection of PAF with duration $\geq 30 \mathrm{~s}$ at 90 days in patients who underwent both ECG monitoring strategies was $16.3 \%$ (7 patients) using patch-based monitoring, as compared to $4.7 \%$ (2 patients) using 24-h Holter monitoring (odds ratio 4.0; 95\% CI 0.8-20.4; $P=0.16$ ). All patients who had newly diagnosed PAF were commenced on anticoagulation therapy by day 90 . No shortterm anticoagulation therapy-related adverse events were recorded by day 90 . There was no difference in the rate of recurrent ischaemic stroke or TIA (patch-based monitoring vs short-duration Holter; 1 (2.3\%) vs 1 (2.1\%); $P=1.00)$ or mortality $[1(2.3 \%)$ vs $0(0 \%) ; P=0.48]$ at 90 days.

\section{Characteristics of patients with atrial fibrillation}

By 90 days of follow-up, among patients in the patchbased monitoring group with PAF detected, the mean time spent in AF in a single day was $4.2 \pm 6.2 \mathrm{~h}$ and the mean value for the maximum time spent in AF was $44.6 \pm 78.6 \mathrm{~h}$. In the patch-based monitoring group, patients with PAF were significantly older than patients with no evidence of PAF, (PAF vs no PAF; $77.4 \pm 6$ vs
$68.6 \pm 14.6 ; P=0.02$ ) (Table 3 ). Patients with PAF also had a history of ischaemic heart disease $(57.1 \%$ vs $11.1 \% ; P=0.02)$, had a higher $\mathrm{CHADS}_{2} \mathrm{VASc}_{2}$ score $(5.0 \pm 0.6$ vs $4.3 \pm 1.2 ; P=0.03)$, had left atrial enlargement $(85.7 \%$ vs $39.2 \% ; P=0.04)$ and had episodes of non-sustained ventricular tachycardia $(71.4 \%$ vs $27.8 \%$; $P=0.04)$.

\section{Economic evaluation}

Implementation of the Zio Patch service at King's College Hospital NHS Foundation Trust would result in 10.8 more strokes avoided per year compared to current practice with Holter monitoring. This would equate to a yearly saving in direct medical costs of $£ 57,481$, increasing to $£ 106,342$ over 5 years. When social care costs are included, incremental savings of $£ 154,716$ can be achieved in the first year and $£ 410,449$ at 5 years. In addition, an analysis of the potential reduction in outpatient follow-up appointment costs resulted in a further saving of $£ 56,149$, giving a total potential saving of $£ 113,630$ over the first year with the use of the ZioPatch service compared to Holter monitoring, increasing to $£ 162,491$ over 5 years. 
Table 1 Baseline characteristics of the study participants

\begin{tabular}{|c|c|c|c|}
\hline Characteristic & $\begin{array}{l}\text { Patch-based monitoring (ACTIVE) } \\
(n=43)\end{array}$ & $\begin{array}{l}\text { Short-duration Holter (CONTROL) } \\
(n=47)\end{array}$ & $P$ value \\
\hline Age, year & $70.7 \pm 12.6$ & $70.0 \pm 13.9$ & 0.82 \\
\hline \multicolumn{4}{|l|}{ Sex, no. (\%) } \\
\hline Male & $26(60.5)$ & $29(61.7)$ & \multirow[t]{2}{*}{1.00} \\
\hline Female & $17(39.5)$ & $18(38.3)$ & \\
\hline \multicolumn{4}{|l|}{ Race or ethnic group, no. (\%) } \\
\hline Asian & $1(2.3)$ & $2(4.3)$ & \multirow[t]{3}{*}{0.80} \\
\hline Black & $11(25.6)$ & $10(21.3)$ & \\
\hline White & $31(72.1)$ & $35(74.5)$ & \\
\hline \multicolumn{4}{|l|}{ Recruitment site, no. (\%) } \\
\hline King's College Hospital & $12(27.9)$ & $22(46.8)$ & \multirow[t]{2}{*}{0.08} \\
\hline Princess Royal University Hospital & $31(72.1)$ & $25(53.2)$ & \\
\hline \multicolumn{4}{|l|}{ Index event, no. (\%) } \\
\hline Stroke & $35(81.4)$ & $43(91.5)$ & \multirow[t]{2}{*}{0.22} \\
\hline $\mathrm{TIA}$ & $8(18.6)$ & $4(8.5)$ & \\
\hline Prior stroke or TIA, no. (\%) & $12(27.9)$ & $7(14.9)$ & 0.20 \\
\hline Score on NIH stroke scale (stroke patients) ${ }^{a}$ & $2.3 \pm 3.7$ & $2.1 \pm 2.6$ & 0.90 \\
\hline Score on $A B C D^{2}$ (TIA patients), no. & $4.1 \pm 0.6$ & $4.2 \pm 0.8$ & 0.87 \\
\hline Hypertension, no. (\%) & $26(60.5)$ & $30(63.8)$ & 0.83 \\
\hline Diabetes, no. (\%) & $10(23.3)$ & $10(21.3)$ & 1.00 \\
\hline $\mathrm{CHADS}_{2} \mathrm{VASC}_{2}$ score, no. ${ }^{\mathrm{b}}$ & $4.4 \pm 1.2$ & $4.3 \pm 1.0$ & 0.61 \\
\hline Ischaemic heart disease, no. (\%) & $8(18.6)$ & $5(10.6)$ & 0.37 \\
\hline Hypercholesterolaemia, no. (\%) & $17(39.5)$ & $17(36.2)$ & 0.83 \\
\hline \multicolumn{4}{|l|}{ Smoking status, no. (\%) } \\
\hline Ex-smoker & $12(27.9)$ & $5(10.6)$ & \multirow[t]{2}{*}{0.27} \\
\hline Current smoker & $6(14)$ & $10(21.3)$ & \\
\hline \multicolumn{4}{|l|}{ Use of antiplatelet, no. $(\%)^{c}$} \\
\hline None & $30(69.8)$ & $33(70.2)$ & \multirow[t]{4}{*}{0.26} \\
\hline Aspirin & $5(11.6)$ & $10(21.3)$ & \\
\hline Clopidogrel & $6(14.0)$ & $4(8.5)$ & \\
\hline Aspirin and Clopidogrel & $2(4.7)$ & $0(0)$ & \\
\hline No. of days from index event to randomisation & $2.0 \pm 1.2$ & $1.9 \pm 1.1$ & 0.60 \\
\hline
\end{tabular}

Plus-minus values are mean \pm SD. $P$ values were calculated with the use of Student's $t$ test, Wilcoxon Mann-Whitney Rank-Sum test or Fisher's exact test, as appropriate. TIA denotes transient ischaemic attack

NIH National Institutes of Health, TIA transient ischaemic attack

a Scores on the National Institutes of Health Stroke Scale range from 0 to 42, with higher scores indicating more severe neurologic deficits. The score was not reported for three patients in the patch-based monitoring (ACTIVE) group and four patients in the 24-h Holter monitoring group

b Scores on the $\mathrm{CHADS}_{2} \mathrm{VASC}_{2}$ risk assessment range from 0 to 6 , with higher scores indicating a greater risk of stroke

c Antiplatelet therapy before the index stroke or TIA

\section{Discussion}

In this randomised trial comparing intermediate-term ECG monitoring by means of a 14-day patch recorder with a conventional short-duration Holter monitor, monitoring with the ZioPatch resulted in a significantly higher rate of detection of PAF, with an associated greater use of anticoagulation, providing strong evidence supporting the adoption of a more prolonged monitoring approach for the detection of PAF in patients with recent cryptogenic stroke or TIA.

\section{Comparison with other cardiac monitoring trials}

The reported incidence of PAF following cryptogenic stroke is wide-ranging from 10 to $30 \%$ [6-8]. This variation can in part be explained by differences in study design, inclusion and exclusion criteria, ECG monitoring strategies and follow-up durations. Our trial observed a $16.3 \%$ incidence of PAF in the patch-based monitoring group at 3 months, which was higher than the $8.9 \%$ incidence observed in the CRYSTAL-AF trial at 6 months [7]. This is likely because CRYSTAL-AF patients already had one 
Table 2 Detection atrial fibrillation and the effect of treatment in the two monitoring groups

\begin{tabular}{|c|c|c|c|c|}
\hline \multirow[t]{2}{*}{ Outcome } & $\begin{array}{l}\text { Patch-based } \\
\text { monitoring (ACTIVE) } \\
(n=43)\end{array}$ & $\begin{array}{l}\text { Short-duration Holter } \\
\text { (CONTROL) } \\
(n=47)\end{array}$ & $P$ value & Odds ratio $(95 \% \mathrm{Cl})$ \\
\hline & \multicolumn{4}{|l|}{ Number (percentage) } \\
\hline \multicolumn{5}{|l|}{ Primary outcome } \\
\hline $\begin{array}{l}\text { Detection of PAF with duration } \geq 30 \text { s at } 90 \text { days (inter- } \\
\text { subject comparison) }\end{array}$ & $7(16.3)$ & $1(2.1)$ & 0.026 & $8.9(1.1-76.0)$ \\
\hline \multicolumn{5}{|l|}{ Secondary outcomes } \\
\hline Detection of PAF with duration $\geq 30$ s at 28 days & $6(14.0)$ & $1(2.1)$ & 0.051 & $7.5(0.9-64.7)$ \\
\hline Anticoagulation therapy use at 90 days & $7(16.3)$ & $1(2.1)$ & 0.026 & $8.9(1.1-76.0)$ \\
\hline Second ischaemic stroke or TIA at 90 days & $1(2.3)$ & $1(2.1)$ & 1.00 & $1.1(0.1-18.1)$ \\
\hline Mortality at 90 days & $1(2.3)$ & $0(0)$ & 0.48 & - \\
\hline
\end{tabular}

PAF paroxysmal atrial fibrillation, TIA transient ischaemic attack

Table 3 Characteristics of the patients with and without atrial fibrillation detected by the patch-based monitoring

\begin{tabular}{|c|c|c|c|}
\hline Characteristic & $\begin{array}{l}\text { Patients with atrial fibrillation } \\
(n=7)\end{array}$ & $\begin{array}{l}\text { Patients without atrial fibrillation } \\
(n=36)\end{array}$ & $P$ value \\
\hline Age, year & $77.4 \pm 6.1$ & $68.6 \pm 14.6$ & 0.02 \\
\hline Male, no. (\%) & $4(57.1)$ & $22(61.1)$ & 1.00 \\
\hline \multicolumn{4}{|l|}{ Index event, no. (\%) } \\
\hline Stroke & $5(71.4)$ & $30(83.3)$ & \multirow[t]{2}{*}{0.60} \\
\hline TIA & $2(28.6)$ & $6(16.7)$ & \\
\hline Prior stroke or TIA, no. (\%) & $2(28.6)$ & $10(27.8)$ & 1.00 \\
\hline Hypertension, no. (\%) & $6(85.7)$ & $20(55.6)$ & 0.22 \\
\hline Diabetes, no. (\%) & $1(14.3)$ & $9(25.0)$ & 1.00 \\
\hline History of ischaemic heart disease, no. (\%) & $4(57.1)$ & $4(11.1)$ & 0.02 \\
\hline \multicolumn{4}{|l|}{ Smoking status, no. (\%) } \\
\hline Ex-smoker & $4(57.1)$ & $8(22.2)$ & \multirow[t]{2}{*}{0.10} \\
\hline Current smoker & $0(0)$ & $6(16.7)$ & \\
\hline $\mathrm{CHADS}_{2}$ VASC $_{2}$ score, no. $(\%)^{\mathrm{a}}$ & $5.0 \pm 0.6$ & $4.3 \pm 1.2$ & 0.03 \\
\hline No. of days from index event to randomisation & $2.3 \pm 2.3$ & $2.0 \pm 1.0$ & 0.73 \\
\hline \multicolumn{4}{|l|}{ Echocardiographic parameters ${ }^{\mathrm{b}}$} \\
\hline Left atrial enlargement ${ }^{c}$ & $6(85.7)$ & $11(39.2)$ & 0.04 \\
\hline Left ventricular ejection fraction (\%) & $53.2 \pm 4.5$ & $53.3 \pm 4.3$ & 0.94 \\
\hline \multicolumn{4}{|l|}{ Arrhythmia detection on patch-based monitoring } \\
\hline Detection of VT & $5(71.4)$ & $10(27.8)$ & 0.04 \\
\hline Number of episodes of VT & $1.1 \pm 0.9$ & $1.3 \pm 3.5$ & 0.81 \\
\hline Detection of VEs & $7(100)$ & $35(97.2)$ & 1.00 \\
\hline Percentage of total beats as VEs & $3.4 \pm 1.7$ & $8.2 \pm 10.5$ & 0.44 \\
\hline Detection of SVES & $7(100)$ & $36(100)$ & - \\
\hline Percentage of total beats as SVEs & $3.1 \pm 1.8$ & $3.0 \pm 2.9$ & 0.97 \\
\hline Detection of SVT & $5(83.3)$ & $27(75.0)$ & 1.00 \\
\hline Number of SVT episodes & $53.2 \pm 63.2$ & $16.4 \pm 25.4$ & 0.22 \\
\hline
\end{tabular}

Plus-minus values are mean \pm SD. $P$ values were calculated with the use of Student's $t$ test, Wilcoxon Mann-Whitney Rank-Sum test or Fisher's exact test, as appropriate. TIA denotes transient ischaemic attack

SVE supraventricular ectopics, SVT supraventricular tachycardia, TIA transient ischaemic attack, VE ventricular ectopics, VT ventricular tachycardia

a Scores on the $\mathrm{CHADS}_{2} \mathrm{VASC}_{2}$ risk assessment range from 0 to 6 , with higher scores indicating a greater risk of stroke

b Transthoracic echocardiography and/or transoesophageal echocardiography was performed in 6 and 28 patients in the PAF and non-PAF groups, respectively

c Left atrial enlargement was defined as left atrial diameter $\geq 40 \mathrm{~mm}$ or left atrial volume index $\geq 29 \mathrm{~mL} / \mathrm{m}^{2}$ 
round of Holter-based cardiac monitoring before inclusion. Likewise, in the EMBRACE trial, where the incidence was $11.6 \%$, patients were recruited after having completed one Holter monitor [8]. Each round of monitoring has eliminated a proportion of PAF from the population.

A notable study difference between EPACS and the above studies is the very early cardiac monitoring, with an average of 2 days after the index stroke or TIA (compared to $\sim 38$ days in the CRYSTAL-AF trial and $\sim 76$ days in EMBRACE trial) before any cardiac monitoring. This makes our study more similar to the recent multicentre FIND-AF trial, which recruited patients within 7 days of the index event. The FIND-AF trial demonstrated that three rounds of 10-day Holter monitoring detected a PAF incidence of $14 \%$ after 6 months compared with 5\% from one 24-h Holter monitor recording [15]. Three Holter ECG's at three different time points over 6 months constitutes a fairly intensive level of cardiac monitoring and achieved comparable detection rates to a single 14-day patch-based monitor, with both being superior to shortduration Holter monitoring. Our study had significant issues in consistently delivering short-duration Holter monitoring compared to delivering patch-based monitoring suggesting that real-world implementation may favour a particular modality regardless of the duration of ECG monitoring [16]. The much earlier extended cardiac monitoring in EPACS offers the opportunity for earlier diagnosis and intervention with anticoagulation for secondary stroke prevention.

\section{PAF detection rate over time following a stroke}

The highest yield for detecting PAF is early following a cryptogenic stroke. In the FIND-AF trial, two-thirds of the PAF events were detected within the first round of 10-day Holter monitoring rather than the subsequent two rounds [15]. In EMBRACE, half of the PAF events were detected within the first week of the 30-day monitoring period, even after patients were enrolled 2.5 months following the index ischaemic event [8]. Similarly, in CRYSTAL-AF, half of the events that were detected within the first 6 months occurred within 42 days of monitoring [7]. The results of these trials suggest that the yield of PAF detection is highest in the first few weeks to months after the ischaemic event. As a result, in addition to the extended monitoring period, the earlier device placement for the patch-based monitoring (2.1 days) compared to the short-duration Holter monitoring (38.9 days), may partially explain the higher AF detection rate observed with the patch-based strategy.

\section{Risk factors for PAF detection}

A number of clinical features that may represent risk factors for a new diagnosis of PAF after cryptogenic stroke or TIA have been studied. We identified that patients in the patch-based monitoring group who had PAF detected were older and were more likely to have a dilated left atrium than those without PAF. Advanced age has been consistently identified as an independent risk factor [17]. An analysis of patients randomised to the implantable recorder found that increased age was the only clinical feature that was independently associated with increased incidence of PAF during follow-up [17]. There is growing evidence for the importance of premature atrial contractions and various echocardiographic parameters including left atrial enlargement $[18,19]$. We found that patients with PAF were also more likely to have a history of ischaemic heart disease. Coronary artery disease is present in greater than $20 \%$ of patients with AF [20]. Whether coronary artery disease per se predisposes patients to AF via atrial ischaemia or whether AF interacts with coronary artery perfusion is uncertain [21]. While higher $\mathrm{CHADS}_{2} /$ $\mathrm{CHADS}_{2} \mathrm{VASc}_{2}$ scores have been associated with an increased risk of AF, this parameter has not been recognised as a significant risk factor in the post-cryptogenic stroke or TIA setting [22]. Patients with PAF were more likely to have higher $\mathrm{CHADS}_{2} \mathrm{VASc}_{2}$ scores in our study. This is unsurprising considering the composite variables in the score are recognised individual risk factors for AF.

\section{Economic evaluation}

While we observed no difference in the rate of recurrent TIA or stroke between the two groups, our study was not powered for this endpoint. Prolonged ECG monitoring with patch-based monitoring was predicted to prevent 10.8 more strokes than short-duration Holter monitoring. This was associated with a yearly saving in direct medical costs of $£ 113,630$, increasing to $£ 162,491$ over 5 years. Based on observed rates of AF detection and anticoagulation from the EMBRACE trial, following a recent cryptogenic stroke or TIA, both 30-day and 14-day ECG monitoring was shown to be cost-effective for preventing recurrent strokes when compared with a repeat 24-h ECG [23]. These results, as well as those from our study, lend support to emerging practice guidelines recommending longer ( $\geq 7$ days) post-stroke ECG monitoring in patients to optimise secondary stroke prevention $[4,5,23]$. 


\section{Limitations}

This study had several limitations. Firstly, the dropout rate was approximately $20 \%$, almost entirely due to Holter ECG service provision. With similar rates of non-compliance observed in the EMBRACE trial, compliance with Holter-based systems for extended monitoring is low, even in the context of a clinical trial. Although compliance rates for the short-duration monitoring were relatively low, the baseline characteristics were similar between the two treatment groups.

Secondly, this study did not directly compare alternative extended monitoring systems like implantable loop recorders. We chose short-duration Holter monitoring as a suitable comparator to the ZioPatch patch-based monitor to reflect current clinical practice ("gold" standard) and for real-world feasibility.

Thirdly, although it is well-established that the detection of AF following a stroke or TIA suggests that there is a casual link between stroke and previously undetected PAF, we acknowledge the possibility that the PAF detected may include the incidence of PAF in the background population as we did not study agematched healthy controls who have not had an ischaemic stroke or TIA. Nonetheless, our incidence rates are much higher than epidemiological studies of AF and it is likely a significant proportion of the PAF we detected were causational to the index event. The REVEAL-AF trial used implantable cardiac monitors to identify an AF detection rate of $6.2 \%$ at 30 days in patients at risk of having an ischaemic stroke [24], which was lower than the incidence detected in our study.

\section{Conclusions}

Our findings have implications for clinical practice, supporting intermediate-term ECG monitoring after a recent cryptogenic embolic stroke or TIA in patients considered appropriate potential candidates for anticoagulation. Common practice of relying on short-term monitoring for the detection of PAF after cryptogenic stroke or TIA is insufficient and should be considered as an initial screen. ZioPatch ${ }^{\circledR}$ patch-based monitoring was well-tolerated and superior to standard practice of short-term ECG monitoring for the detection of PAF in patients with a cryptogenic stroke or TIA. Future, appropriately powered, studies are required to demonstrate that the increased PAF detection through extended ECG monitoring results in reduced recurrent stroke or TIA rates, thereby justifying the cost-effectiveness of using more extensive, invasive or longerterm ECG monitors.

\section{Additional file}

Additional file 1. Probability and cost assumptions for economic model

\section{Abbreviations}

AF: atrial fibrillation; ECG: electrocardiogram; EPACS: early prolonged ambulatory cardiac monitoring in stroke; Cl: confidence interval; PAF: paroxysmal atrial fibrillation; SVE: supraventricular ectopics; SVT: supraventricular tachycardia; TIA: transient ischaemic attack; VE: ventricular ectopics; VT: ventricular tachycardia.

\section{Acknowledgements}

We would like to thank all the patients who volunteered to participate in this trial and the research assistants for their contribution throughout this trial. We would also like to thank Judy Lenane, Chief Clinical Officer of iRhythm Technologies (USA); Jennifer Weller and Lee Cochrane of iRhythm Technologies (UK) for their assistance in this study.

\section{Authors' contributions}

JT and BPJ researched the literature and conceived the study. JT and BPJ were involved in protocol development and gaining ethical approval. LS, FKC, JAT, BPJ and JT were involved in patient recruitment. AK, NG and JT performed the data analysis. AK wrote the first draft of the manuscript. All authors read and approved the final manuscript.

\section{Funding}

This work was supported by an investigator-initiated research Grant from Bristol-Myers Squibb-Pfizer alliance (Grant Number CV185-475).

\section{Availability of data and materials}

The datasets generated and/or analysed during the current study are not publicly available due to ethical restrictions but are available from the corresponding author on reasonable request.

\section{Ethics approval and consent to participate}

NRES Committee London approved this study (REC Number: 15/LO/1534).

Written informed consent was obtained from all subjects before the study.

\section{Competing interests}

JT has received grants from Bristol-Meyers-Squibb; Goldman Sachs, Michael J Fox Foundation for Parkinson's disease.

\section{Author details}

${ }^{1}$ King's College London NHS Foundation Trust, King's College Hospital, Denmark Hill, London SE5 9RS, UK. ${ }^{2}$ Imperial College Healthcare NHS Trust, Hammersmith Hospital, Du Cane Road, London W12 OHS, UK.

Received: 12 February 2019 Accepted: 12 July 2019

Published online: 26 July 2019

\section{References}

1. Li L, Yiin GS, Geraghty OC, Schulz UG, Kuker W, Mehta Z, et al. Oxford Vascular Study. Incidence, outcome, risk factors, and long-term prognosis of cryptogenic transient ischaemic attack and ischaemic stroke: a population-based study. Lancet Neurol. 2015;14:903-13.

2. Sacco RL, Ellenberg JH, Mohr JP, Tatemichi TK, Hier DB, Price TR, et al. Infarcts of undetermined cause: the NINCDS Stroke Data Bank. Ann Neurol. 1989;25:382-90.

3. Jabaudon DJ, Sztajzel J, Sievert K, Landis T, Sztajzel R. Usefulness of ambulatory 7-day ECG monitoring for the detection of atrial fibrillation and flutter after acute stroke and transient ischemic attack. Stroke. 2004;35:1647-51. 
4. Culebras A, Messé SR, Chaturvedi S, Kase CS, Gronseth G. Summary of evidence-based guideline update: prevention of stroke in nonvalvular atrial fibrillation: Report of the Guideline Development Subcommittee of the American Academy of Neurology. Neurology. 2014;82:716-24.

5. Party Intercollegiate Stroke Working. National clinic guideline for stroke. 5th ed. London: Royal College of Physicians; 2016.

6. Rizos T, Guntner J, Jenetzky E, Marquardt L, Reichardt C, Becker R, et al. Continuous stroke unit electrocardiographic monitoring versus 24-hour Holter electrocardiography for detection of paroxysmal atrial fibrillation after stroke. Stroke. 2012;43:2689-94.

7. Sanna T, Diener HC, Passman RS, Di Lazzaro V, Bernstein RA, Morillo CA, et al. CRYSTAL AF Investigators. Cryptogenic stroke and underlying atrial fibrillation (CRYSTAL AF). N Engl J Med. 2014;370:2478-86.

8. Gladstone DJ, Spring M, Dorian P, Panzov V, Thorpe KE, Hall J, et al. EMBRACE investigators and coordinators. EMBRACE Investigators and Coordinators. Atrial fibrillation in patients with cryptogenic stroke. N Engl J Med. 2014;370:2467-77.

9. Turakhia MP, Hoang DD, Zimetbaum P, Miller JD, Froelicher VF, Kumar UN, et al. Diagnostic utility of a novel leadless arrhythmia monitoring device. Am J Cardiol. 2013;112:520-4.

10. Rosenberg MA, Samuel M, Thosani A, Zimetbaum PJ. Use of a noninvasive continuous monitoring device in the management of atrial fibrillation: a pilot study. Pacing Clin Electrophysiol. 2013;36:328-33.

11. Barrett PM, Komatireddy R, Haaser S, Topol S, Sheard J, Encinas J, et al. Comparison of 24-hour Holter monitoring with 14-day novel adhesive patch electrocardiographic monitoring. Am J Med. 2014;127(1):95.e11-7.

12. The Information Centre for Health and Social Care. Hospital episode statistics-HESonline. http://www.hesonline.nhs.uk. Accessed 10 Nov 2018.

13. NHS Improvement. NHS Reference Costs 2016-2017. https://impro vement.nhs.uk/resources/reference-costs/. Accessed 10 Nov 2018.

14. Xu XM, Vestesson E, Paley L, Desikan A, Wonderling D, Hoffman A, et al. The economic burden of stroke care in England, Wales and Northern Ireland: using a national stroke register to estimate and report patient-level health economic outcomes in stroke. Eur Stroke J. 2018;3(1):82-91.

15. Wachter R, Gröschel K, Gelbrich G, Hammann GF, Kermer P, Liman J, et al. Find-AF(randomised) Investigators and Coordinators. Holter-electrocardiogram-monitoring in patients with acute ischaemic stroke (Find-AFRANDOMISED): an open-label randomised controlled trial. Lancet Neurol. 2017;16:282-90.
16. Levin $L \AA$, Husberg $M$, Sobocinski PD, Kull VF, Friberg $L$, Rosenqvist $M$, et al. A cost-effectiveness analysis of screening for silent atrial fibrillation after ischaemic stroke. Europace. 2015;17:207-14.

17. Thijs VN, Brachmann J, Morillo CA, Passman RS, Sanna T, Bernstein RA, et al. Predictors for atrial fibrillation detection after cryptogenic stroke: results from CRYSTAL AF. Neurology. 2016;86:261-9.

18. Gladstone DJ, Dorian P, Spring M, Panzov V, Mamdani M, Healey JS, et al. EMBRACE Steering Committee and Investigators. Atrial premature beats predict atrial fibrillation in cryptogenic stroke: results from the EMBRACE trial. Stroke. 2015;46:936-41.

19. Yaghi S, Moon YP, Mora-MCLaughlin C, Willey JZ, Cheung K, Di Tullio MR, et al. Left atrial enlargement and stroke recurrence: the Northern Manhattan Stroke Study. Stroke. 2015;46:1488-93.

20. Nieuwlaat R, Capucci A, Camm AJ, Olsson SB, Andresen D, Davies DW et al. European Heart Survey Investigators. Atrial fibrillation management: a prospective survey in ESC member countries: the Euro Heart Survey on Atrial Fibrillation. Eur Heart J. 2005;26:2422-34

21. Motloch LJ, Reda S, Larbig R, Wolff A, Motloch KA, Wernly B, et al. Characteristics of coronary artery disease among patients with atrial fibrillation compared to patients with sinus rhythm. Hellenic J Cardiol. 2017;58:204-12.

22. Tischer T, Schneider R, Lauschke J, Nesselmann C, Klemm A, Diedrich D, et al. Prevalence of atrial fibrillation in patients with high CHADS2- and CHA2DS2VASC-scores: anticoagulate or monitor high-risk patients? Pacing Clin Electrophysiol. 2014;37:1651-7.

23. Yong JH, Thavorn K, Hoch JS, Mamdani M, Thorpe KE, Dorian P, et al. Potential cost-effectiveness of ambulatory cardiac rhythm monitoring after cryptogenic stroke. Stroke. 2016;47(9):2380-5.

24. Reiffel JA, Verma A, Kowey PR, Halperin JL, Gersh BJ, Wachter R, et al. REVEAL AF Investigators. Incidence of previously undiagnosed atrial fibrillation using insertable cardiac monitors in a high-risk population: the REVEAL AF study. JAMA Cardiol. 2017;2(10):1120-7.

\section{Publisher's Note}

Springer Nature remains neutral with regard to jurisdictional claims in published maps and institutional affiliations.
Ready to submit your research? Choose BMC and benefit from:

- fast, convenient online submission

- thorough peer review by experienced researchers in your field

- rapid publication on acceptance

- support for research data, including large and complex data types

- gold Open Access which fosters wider collaboration and increased citations

- maximum visibility for your research: over $100 \mathrm{M}$ website views per year

At BMC, research is always in progress.

Learn more biomedcentral.com/submissions 